\title{
Caracterización del "veranillo" en dos cuencas de la vertiente del Pacífico de Costa Rica, América Central
}

\section{Eric J. Alfaro ${ }^{1,2,3}$}

1. Centro de Investigaciones Geofísicas, 2. Escuela de Física y 3. Centro de Investigación en Ciencias del Mar y Limnología, Universidad de Costa Rica, 11501-2060 San José, Costa Rica; erick.alfaro@ucr.ac.cr

\author{
Recibido 26-VIII-2013. C Corregido 12-II-2014. Aceptado 27-IX-2014.
}

\begin{abstract}
Characterization of the Mid Summer Drought in two Pacific slope river basins of Costa Rica, Central America. The Eastern Tropical Pacific region is characterized by climate features rarely observed in tropical regions, one of them is the Mid-Summer Drought (MSD), "veranillo" or "canícula" in Spanish. On the Pacific slope of Central America, the annual precipitation cycle is characterized by two rainfall maxima in June and September-October, an extended dry season from November to May, and a shorter reduced precipitation period during July-August (MSD), during July, the magnitude of the trade winds increases and this is associated also with the Caribbean Low Level Jet (CLLJ), but characterization of these features using monthly data is difficult. In this work, seven daily gauge stations records, located at two important river basins of Costa Rica, Tarcoles and Tempisque, were studied to characterize the MSD from 1937 to 2012. Among the aspects considered are the MSD Start, Timing, End, Duration, Intensity and Magnitude. The modulation and seasonal predictability of these aspects by climate variability sources as Equatorial Eastern Pacific was lately explored, showing that warmer (cooler) conditions in Niño 3.4 tend to be associated to drier (wetter) MSD events. Rev. Biol. Trop. 62 (Suppl. 4): 1-15. Epub 2014 Diciembre 01.
\end{abstract}

Key words: Mid Summer Drought, climate variability, Tempisque, Tarcoles, seasonal climate prediction, categorical analysis, Central America.

Una de las primeras caracterizaciones del veranillo en Costa Rica la realizó Ramírez (1983). Esta autora describió que la estación lluviosa en la vertiente del Pacífico de Costa Rica, que normalmente se extiende de mayo a mediados de noviembre, tiene un receso en los meses de julio y agosto que se conoce como "veranillo", debido al hecho de que en medio de la estación lluviosa se dan condiciones similares a la estación seca, que suele llamarse popularmente "verano" en el país (típicamente de mediados de noviembre a finales de abril). Estas condiciones son: vientos alisios fuertes durante todo el día en la mayor parte del país, disminución de la precipitación y la humedad relativa en la vertiente del Pacífico, con la aparición de días secos consecutivos, que coincide con un máximo de precipitación sobre la vertiente del Caribe. Sin embargo, y a pesar que el periodo de disminución de las lluvias se presenta casi todos los años, este es muy variable de un año a otro en aspectos como su inicio y duración, lo cual es un factor de incertidumbre para diferentes sectores socio-económicos como el agrícola, esta variabilidad fue notada luego también por Amador (2008).

Posteriormente, Magaña, Amador y Medina (1999), así como Amador, Alfaro, Lizano y Magaña (2006), mostraron que la señal del veranillo se observa en una región bastante más amplia del Pacífico Tropical del Este (PTE) y que durante la transición de una actividad convectiva intensa a débil (débil a intensa), los vientos alisios se fortalecen (debilitan). Esta aceleración en el flujo alisio es parte de la respuesta dinámica de la atmósfera en niveles 
bajos por la magnitud del forzamiento sobre la convección en la ZCIT. La intensificación de los vientos alisios durante julio y agosto, junto con el forzamiento orográfico de las montañas en buena parte de América Central, provocan un máximo de precipitación a lo largo de la costa del Caribe y un mínimo en la del Pacífico. Amador et al. (2006), muestran que durante el mes de julio se alcanza un fuerte gradiente meridional en la presión superficial entre latitudes medias y altas en el Atlántico. Cabe destacar también, que es durante el mes de julio que la Corriente en Chorro de Bajo Nivel del Caribe alcanza su máximo (Amador, 2008). Recientemente Nakaegawa, Kitoh, Ishizaki, Kusunoki y Murakami (2013), encontraron que el flujo de humedad del este asociado a esta corriente, podría incrementarse hacia final del siglo XXI por efectos del cambio climático, sin embargo, no encontraron resultados consistentes en cuanto a la intensificación o debilitamiento en la magnitud de la corriente.

Magaña et al. (1999) explican que la evolución del veranillo se puede entender a través de los cambios en la divergencia (convergencia) de los vientos en bajo nivel sobre la piscina de agua cálida ubicada al oeste de la costa pacífica del sur de México y América Central (Wang \& Enfield, 2003). La máxima actividad convectiva en el norte del PTE, cuando se establece la estación lluviosa, se alcanza cuando las temperaturas superficiales del mar (TSM) exceden $\operatorname{los} 29^{\circ} \mathrm{C}$ en mayo. Posteriormente, la TSM en esta región oceánica decrece alrededor de $1^{\circ} \mathrm{C}$, debido a la disminución en la incidencia de radiación solar y al fortalecimiento de los vientos del este durante julio y agosto. Esta reducción a $28^{\circ} \mathrm{C}$ produce una disminución sustancial de la actividad convectiva, asociada con interacciones no lineales entre la TSM y la convección tropical (Magaña et al., 1999). Esta disminución de la convección tropical, hace que aumente la radiación solar incidente y que aumente la TSM que alcanza un segundo máximo de alrededor de $28,5^{\circ} \mathrm{C}$ a finales de agosto y principios de setiembre. Este segundo incremento de la TSM provoca una convergencia fuerte en bajo nivel que refuerza la convección y se refleja en un segundo máximo de precipitación en el PTE. Esta señal del veranillo puede detectarse también en otras regiones como la cuenca del Mar Caribe o el Norte de Suramérica (Magaña et al., 1999; Taylor, Enfield \& Chen, 2002; Amador et al., 2006), sin embargo el modelo conceptual que explica la señal en el PTE, no es directamente aplicable para estos casos.

Más recientemente, Karnauskas, Giannini, Seager y Busalacchi (2013), estudiaron la distribución global, la evolución estacional y los mecanismos asociados con el veranillo, usando un conjunto de datos de alta resolución espacial y temporal de estaciones y de reanálisis, haciendo énfasis en la costa pacífica de América Central y del sur de México. Ellos encontraron también que hay otras regiones del globo con características similares al veranillo y de diferentes magnitudes. Para explicar el veranillo, ellos proponen un mecanismo que relaciona la dependencia latitudinal de los dos máximos de precipitación con el cruce bianual de la declinación solar, lo cual provoca dos picos de inestabilidad convectiva y de precipitación. Este mecanismo propuesto por Karnauskas et al. (2013), difiere del de Magaña et al. (1999) basado en retroalimentaciones positivas y negativas que favorecen o no la precipitación, ya que más bien propone un modelo basado en dos retroalimentaciones positivas que favorecen la lluvia en dos épocas del año. Sin embargo, al igual que Magaña et al. (1999), ellos notaron que además del veranillo, varios procesos remotos y relacionados, también alcanzan su máximo en esta época del año, como son la actividad del monzón de América del Norte, la Corriente en Chorro de Bajo Nivel del Caribe y la Alta subtropical del Atlántico Norte. Esto no favorece la lluvia a lo largo de la costa pacífica de América Central y genera además variabilidad interanual en la magnitud y en la fecha del veranillo, tal y como lo notó previamente Amador (2008).

En Costa Rica, Ramírez (1983) encontró que dos de las regiones en donde está muy bien definido el veranillo y en donde es más intenso son la parte central del Pacífico Norte y la parte 
baja del Valle Central de Costa Rica, al oeste del mismo, en donde se ubican las cuencas de los ríos Tempisque y Grande de Tárcoles, dos de las más importantes del país. En esta última región, la secuencia más probable de días secos es de dos $(70 \%)$. Se observa también que en estas regiones, el descenso de la lluvia se acentúa a partir del 1 de julio y que la precipitación suele aumentar de nuevo luego de los primeros 15 días de agosto.

Espinoza y Villalta (2004) describen que la cuenca del Río Grande Tárcoles se ubica en el sector central y occidental del Valle Central de Costa Rica y se extiende hasta la vertiente del Pacífico (Fig. 1a), abarcando parcialmente cinco de las siete provincias del país y 36
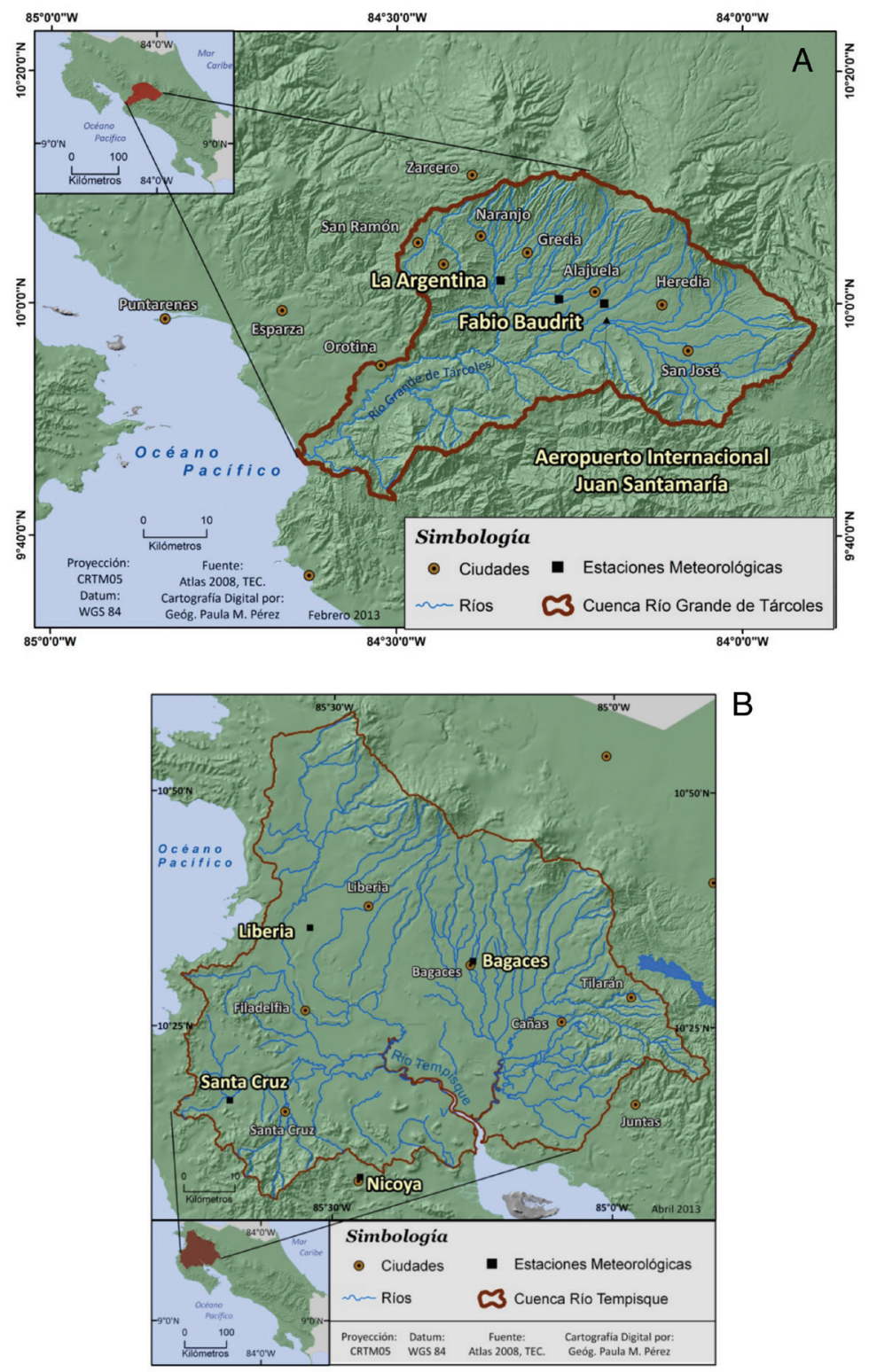

B

Fig. 1. Estaciones usadas en la cuenca del a) Grande de Tárcoles y b) Tempisque.

Fig. 1. Stations used at a) Grande de Tarcoles and b) Tempisque river basin. 
municipios de los 81 existentes. Tiene un área de $2155,5 \mathrm{~km}^{2}$ lo que representa el $4.2 \%$ de la superficie nacional. Esta cuenca presenta las siguientes características: i) concentra la mayor parte de la población del país, 2298617 habitantes, lo cual equivale a cerca de, un $55 \%$ de la población nacional (INEC, 2012); ii) alberga las principales actividades productivas y económicas de Costa Rica en donde por ejemplo se ubica el $80 \%$ de las industrias, incluyendo las de alta tecnología, de bebidas, químicas, agroindustriales, metalúrgicas; así como el principal comercio y la mayor prestación de servicios del país; iii) se procesa más del $50 \%$ de la producción de café y la actividad agrícola y pecuaria ocupa un lugar preponderante; iv) cinco represas para producción eléctrica se encuentran en esta región (Puente de Mulas, Belén, Virilla, La Garita y Nuestro Amo); v) la mayor parte de la población se ubica en la parte media de la cuenca, en el sector central oriental; vi) es la más contaminada del país y una de las mayores en el nivel centroamericano; recibe aproximadamente el $67 \%$ de la carga orgánica del país. Se puede afirmar que la situación más crítica en la cuenca la representan las aguas superficiales; sin embargo, se conoce que las aguas subterráneas están siendo fuertemente amenazadas por la expansión urbana y agrícola. Ha empezado a tener problemas de contaminación especialmente por nitratos producto del uso fertilizantes en los cultivos de café, también por la percolación de las aguas negras provenientes de la utilización intensiva de más de 200000 tanques sépticos, así como por la filtración de aguas contaminadas a los mantos acuíferos. Las fuentes de contaminación más importantes son las descargas de aguas residuales producidas por los principales prestadores de servicios públicos de la cuenca. Puede observarse que existe un problema de contaminación de las aguas superficiales, que se manifiesta a lo largo de toda la cuenca y que produce efectos negativos para todos los usos del agua, tales como: consumo humano, riego, turismo, recreación, paisaje y estética. Espinoza y Villalta (2004) concluyen que los problemas de contaminación de las aguas superficiales y subterráneas de la cuenca del Tárcoles son sumamente graves, con potencial de impactar negativamente la calidad de vida de los costarricenses a través de su desarrollo ambiental, económico y social. A la contaminación de las aguas se ha adicionado la deposición de desechos sólidos en los cauces de los ríos, los cuales han sido utilizados como basureros colectivos. Asimismo, se agregan las consecuencias de la explotación de tajos, tales como la utilización de los cuerpos de agua para depositar materiales de destape y rechazo, producto de estas operaciones. Por lo tanto, el problema de la contaminación de la Cuenca del Tárcoles está claramente identificado, así como su impacto negativo en la calidad de vida de los pobladores de la cuenca y en el desarrollo económico, social y ambiental.

Por su parte, Echeverría-Bonilla (2004) describe que la cuenca del río Tempisque está localizada al Noroeste de Costa Rica, en la provincia de Guanacaste, Costa Rica (Fig. 1b). Tiene una extensión de $3407 \mathrm{~km}^{2}$ (aproximadamente el $34 \%$ de la provincia y el $7 \%$ del territorio nacional) y en la cuenca los principales cultivos son actualmente caña de azúcar, arroz (cerca de un $25 \%$ de la producción nacional) y es la mayor zona productora de melón para exportación. Tiene importantes complejos agroindustriales y se encuentran importantes ecosistemas, como los humedales de Bolsón, Riberino Zapandí, Palo Verde, algunos hábitats como: bosques secos de bajura, sabanas arboladas y bosques siempre verdes, incluyendo algunas de estas áreas dentro de parques nacionales. A partir de la última década, ha sido también fuente de agua para actividades turísticas cercanas a la costa, como el Proyecto Turístico Papagayo. La precipitación promedio es de $1833 \mathrm{~mm}$. Mientras que la cuenca presenta exceso de agua en la época lluviosa que incluso provoca graves y recurrentes inundaciones, en la época seca la disponibilidad del recurso disminuye sustancialmente, con prolongaciones de hasta seis meses. Lo anterior hace según EcheverríaBonilla (2004), que alrededor del agua de la cuenca y en la zona de influencia de la misma, se desenvuelva una gran cantidad de actores 
con múltiples y diversos intereses: productores agropecuarios e industriales; instituciones públicas; municipios; proyectos de desarrollo turístico, comunidades con aspiraciones de crecimiento económico como Liberia, Filadelfia y Santa Cruz; y diversas organizaciones no gubernamentales, conocidas como ONGs. El agua es clave para la producción en esta zona estacionalmente seca, por lo que hay grandes efectos económicos, sociales y ambientales ligados a la distribución, acceso y uso de la misma. Por esta razón, existen grandes preocupaciones por parte de algunos sectores de usuarios, institucionales y no gubernamentales, respecto a la disponibilidad del recurso y la demanda del mismo. En la actualidad, en la cuenca de río Tempisque, excepto en tiempos y espacios geográficos muy puntuales, no se considera que exista un problema real de escasez de agua, pero sí se detectan graves problemas en la gestión del agua. Las principales fallas en la gestión de los recursos hídricos incluyen la incorrecta aplicación de la normativa, competencias poco claras de las instituciones, el desconocimiento real del potencial hídrico de la cuenca y un precio excesivamente bajo del agua. Esto se traduce en conflictos por el uso del agua, concesiones en exceso de la disponibilidad y un incremento en los niveles de contaminación de los ríos y otras aguas.

Debido a la importancia del manejo del recurso hídrico en dos de las principales cuencas de Costa Rica, como lo son Grande de Tárcoles y Tempisque, es que se plantea como objetivo de este trabajo la caracterización del veranillo en estas regiones y su posible predicción estacional. Al contar con herramientas de análisis objetivo, estas pueden ayudar a la solución de problemas, ya que Espinoza y Villalta (2004) señalan que es conveniente tomar decisiones y acciones, en el marco de la Gestión Integrada de los Recursos Hídricos. Además, la aplicación de herramientas de pronóstico en una época de disminución del recurso como el veranillo, ayudaría en el manejo del mismo y según Echeverría-Bonilla (2004) redundaría en que el agua se convierta en un elemento para el desarrollo económico y social, y no en un elemento que limita el mismo en la cuenca hidrográfica.

\section{MATERIALES Y MÉTODOS}

Se usaron los registros de precipitación de siete estaciones pluviométricas con registros acumulados diarios. Tres de estas estaciones se ubicaron en la cuenca del río Grande de Tárcoles al oeste del Valle Central. Los nombres de estas estaciones son La Argentina, Juan Santamaría y Fabio Baudrit (Fig. 1a). En el orden anterior, el periodo de disponibilidad de datos en estas estaciones se ubicó entre los años 1937-2010, 1956-2012 y 1959-2012, con un porcentaje de datos faltantes de 4,6, 0,9 y 5,1, respectivamente. Lo anterior permitió el estudio y caracterización del veranillo en esta cuenca entre los años 1937 y 2012, excepto para los años 1949 y 1950 debido a la falta de datos para hacerlo, es decir 74 eventos. Las estaciones se escogieron al oeste del valle ya que una de las principales causas que puede afectar el análisis según Ramírez (1983) es el derrame de lluvia desde la vertiente Caribe debido al incremento del viento durante esta época el año (Amador, 2008), asociado con la corriente en chorro de bajo nivel del Caribe.

Para la caracterización del veranillo en la cuenca del río Tempisque se usaron las estaciones llamadas Bagaces, Liberia, Santa Cruz y Nicoya (Fig. 1b). Las mismas tenían registros disponibles para los años 1974-2007, 19572010, 1937-2010 y 1949-2010, respectivamente. En este mismo orden el porcentaje de datos faltantes fue de 1,0, 13,8, 22,2 y 6,7. Los registros permitieron la caracterización del veranillo en esta cuenca entre los años 1949 y 2010, además del año 1937, es decir 63 eventos.

Como primer paso, los datos faltantes de los registros diarios se rellenaron con un modelo autorregresivo de orden 1 , de acuerdo a la metodología descrita por Alfaro y Soley (2009). Para minimizar las falsas interrupciones de los eventos de veranillo debido a las perturbaciones que pueden afectar el análisis de variabilidad temporal de menos de un mes, como el debilitamiento del flujo alisio, el 
acercamiento de la Zona de Confluencia Intertropical (por presencia de bajas presiones en el Mar Caribe o en el PTE) y el derrame de lluvia desde la vertiente Caribe, descritas en detalle por Ramírez (1983), se filtraron las series de tiempo con una media móvil triangular de 31 datos de acuerdo a Soley (1994). Luego, se calculó el promedio aritmético de todas las series ubicadas en una misma cuenca para producir un índice de precipitación. Utilizando este índice, se examinó los meses de mayo a setiembre para encontrar el mínimo de precipitación en ese periodo, el cual se tomó como la fecha del mínimo de precipitación del evento del veranillo durante ese año en particular. Para cada año, la fecha del inicio del evento se definió como el día en donde el índice empieza a disminuir y la del final de cada uno cuando esta serie deja de aumentar. La duración del mismo se estimó como la fecha del final menos la del inicio en días julianos. La intensidad de cada evento se asoció a la precipitación promedio diaria durante los eventos y la magnitud de cada uno de ellos se definió como el valor de precipitación localizado en el mínimo del valle de cada evento en particular. La Fig. 2 muestra como ejemplo la caracterización del año 1965 en la cuenca del Grande de Tárcoles. De acuerdo a esta figura, el evento del año 1965 inició el 22 de junio y finalizó el 2 de agosto, para una duración de 42días. El mínimo se ubicó el 14 de julio con una precipitación asociada de $1,9 \mathrm{~mm}$ (magnitud). La precipitación promedio (intensidad) estimada entre las fechas del inicio y final fue de $5,2 \mathrm{~mm} \mathrm{día}^{-1}$.

Del procedimiento anterior, se obtuvieron cinco series anuales para cada cuenca y se estudió su relación con la variabilidad climática asociada con El Niño-Oscilación del Sur (ENOS). Para ello se emplearon las anomalías del índice Niño 3.4 observadas durante el mes de junio. Se usaron dos herramientas para explorar estas relaciones. La primera fue el análisis de Tablas de Contingencia propuesto por Alfaro et al. (2003), en donde tanto la serie anual asociada al veranillo, así como la del índice Niño 3.4 se categorizaron en tres clases, a saber, Bajo lo Normal (BN), Neutral (N) y Arriba de lo Normal (AN), para construir tablas de $3 \times 3$ de 9 categorías conjuntas. Los límites de clases se establecieron de acuerdo a los percentiles 33,33 y 66,67 en ambas series.

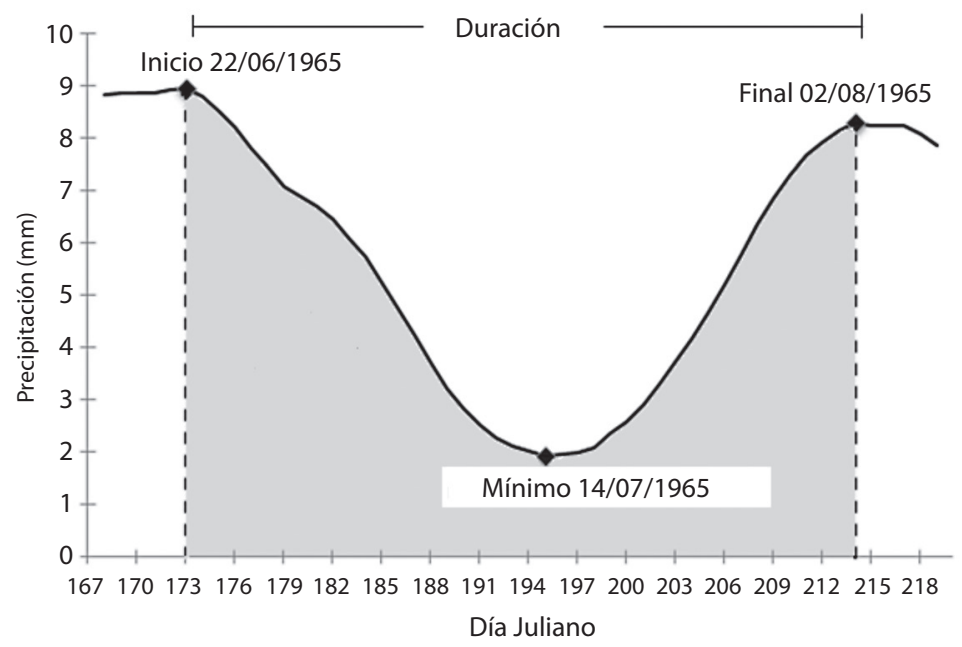

Fig. 2. Caracterización del veranillo para el año 1965 en la cuenca del Grande de Tárcoles. De acuerdo a esta figura, el evento del año 1965 inició el 22 de junio y finalizó el 2 de agosto, para una duración de 42días. El mínimo se ubicó el 14 de julio con una magnitud de $1.9 \mathrm{~mm}$ y una intensidad de $5.2 \mathrm{~mm} \mathrm{día}{ }^{-1}$.

Fig. 2. Characterization of the 1965 Mid Summer Drought event at the Grande de Tarcoles basin. According to the figure, this event started in June $22^{\text {nd }}$ and ended by August 2, with duration of 42days. Its minimum was located in July 14 , with a magnitude of $1.9 \mathrm{~mm}$ and an event intensity of $5.2 \mathrm{~mm} \mathrm{day}^{-1}$. 
La segunda herramienta estadística correspondió a los modelos logit, construidos de acuerdo a lo explicado por Wilks (2006), de acuerdo a la ecuación

(Ec. 1)

$$
y(x)=\frac{1}{1+e^{-\left(\beta_{0}+\beta_{1} x\right)}}
$$

Para el ajuste de la ecuación anterior, el valor de $x$ corresponde al valor de la anomalía del índice Niño 3.4 observada durante el mes de junio y $y$ es alguna de las seis series binarias anuales sobre algún aspecto del veranillo, con valores de 1 asociados a la categoría $\mathrm{AN}$ o $\mathrm{BN}$, según sea el caso, y 0 de otra forma. Nótese que este ajuste da como resultado dos series de probabilidad anuales $\left(y_{\mathrm{AN}} \mathrm{y} y_{\mathrm{BN}}\right)$ y se puede calcular una tercera asociada a la categoría $\mathrm{N}$ estimada como $y_{\mathrm{N}}=1-y_{\mathrm{AN}}-y_{\mathrm{BN}}$. Las mismas se pueden multiplicar por 100 para expresarlas como porcentajes, es decir

(Ec. 2)

$$
y_{\mathrm{BN}}+y_{\mathrm{N}}+y_{\mathrm{AN}}=100,
$$

para todos los valores de las anomalías observadas del índice Niño 3.4 y durante todos los años que comprendió el estudio.

El índice Niño 3.4 se obtuvo del repositorio del Instituto Internacional de Investigación para el Clima y la Sociedad (IRI, por sus siglas en inglés) (Consultado: 24 de junio de 2013, http://iridl.ldeo.columbia.edu/SOURCES/.Indices/.nino/.EXTENDED/.NINO34/ $\mathrm{T}+$ exch+table-+text+text+skipany $\mathrm{NaN}+$ table+.html).

Por último, se consultaron los Boletines Meteorológicos Mensuales producidos por el Instituto Meteorológico Nacional (IMN) de Costa Rica para extraer los impactos climáticos de estos eventos del veranillo. Los años consultados fueron 1977, 1982, 1986, 1993, 2000, 2001, 2003, 2009 y 2012, ubicados en la categoría $\mathrm{BN}$ de las series anuales de magnitud o intensidad en alguna de las cuencas. Cabe destacar que la información de eventos anteriores no se pudo recolectar, debido a que no se encontraron Boletines Meteorológicos para esos años anteriores a 1977. Las categorías principales usadas para agrupar estos impactos fueron inundaciones, déficit de lluvias, aguaceros fuertes, temperaturas máximas por encima o por debajo del promedio, vientos fuertes, deslizamientos y daños varios.

\section{RESULTADOS}

La Fig. 3 muestra los resultados obtenidos para las cinco variables consideradas del veranillo en la cuenca del Tárcoles. De acuerdo al ámbito intercuartil $\left(\mathrm{Q}_{3}-\mathrm{Q}_{1}\right)$, se observa de la Fig. 3a que el inicio de los eventos tiende a darse entre 14 de junio y el 17 de julio, su mínimo entre el 7 de julio y el 4 de agosto y el final entre el 26 de julio y el 23 de agosto, en donde el único año considerado atípico fue el de 1989 con un final muy temprano del evento. Nótese que la variabilidad observada entre las fechas de los eventos fue muy alta, lo que concuerda con lo observado por Ramírez (1983) y Amador (2008). El 50\% de las observaciones de la duración de los eventos se ubicaron en los 30 y 48días, las intensidades de los eventos se observaron entre 6,1 y $8,6 \mathrm{~mm}_{\text {día }}{ }^{-1}$ y entre 2,7 y $5,2 \mathrm{~mm}$ para las magnitudes de los mismos (Figs. 3b y c). Dentro de los años que presentaron condiciones más secas al tomar en cuenta estos dos últimos aspectos están 1942, 1969, 1972, 1977, 1982, 1986, 1993, 2001, 2003 y 2009. El análisis también mostró al año 2012 como el evento de mayor duración (Figs. 3d).

Los resultados correspondientes a la cuenca del Tempisque se muestran en la Fig. 4. Se nota de la Fig. 4a que el inicio de los eventos tiende a darse entre 11 de junio y el 21 de julio, su mínimo entre el 9 de julio y el 4 de agosto y el final entre el 28 de julio y el 30 de agosto, valores muy similares a aquellos mostrados en la Fig. 3a. El 50\% de las observaciones de la duración de los eventos se ubicaron en los 34,5 y 54días, las intensidades de los eventos se observaron entre 4,3 y $8,5 \mathrm{~mm} \mathrm{día}^{-1}$ y entre 2,0 y $3,9 \mathrm{~mm}$ para las magnitudes de los mismos (Figs. 4b, c y d), valores en general menores a 

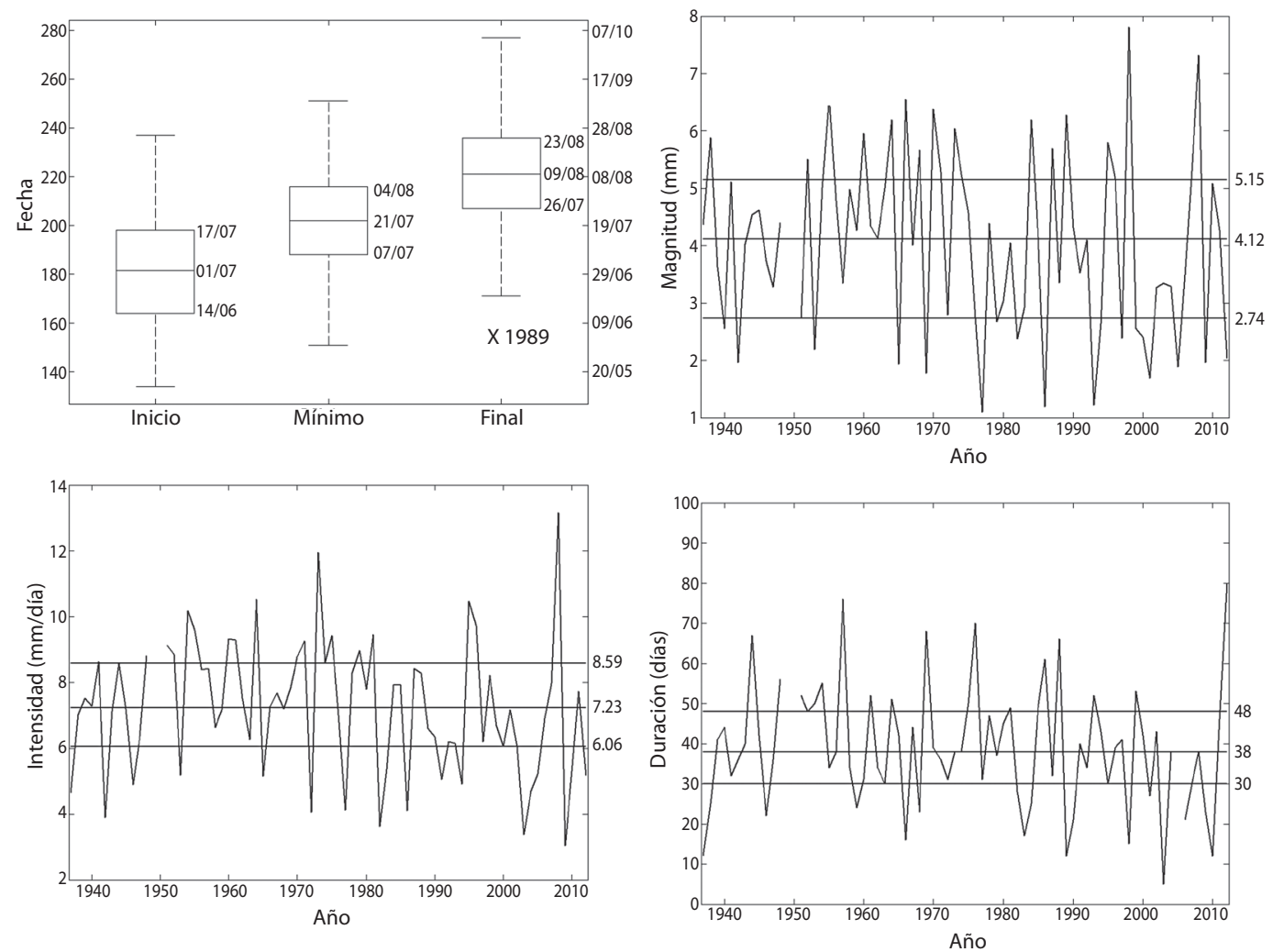

Fig. 3. Características del veranillo en la cuenca del Grande de Tárcoles. a) Gráficos de cajas para el inicio, la fecha del mínimo y el final de los eventos. Los bigotes se calcularon como $Q_{3}+1.5 *\left(Q_{3}-Q_{1}\right)$ y $Q_{1}-1.5 *\left(Q_{3}-Q_{1}\right)$, con $Q_{1}$ y $Q_{3}$ el primer y tercer cuartil, respectivamente. En el eje $y$ se dan las fechas julianas (izquierda) y gregorianas (derecha). b) Intensidad, c) Magnitud y d) Duración de los eventos, las líneas horizontales son para los cuartiles $\mathrm{Q}_{1} \mathrm{Q}_{2}$ (mediana) y $\mathrm{Q}_{3}$.

Fig. 3. Characteristics of the Mid Summer Drought at Grande de Tarcoles basin. a) Box plots for the Star, Timing and End of the events. Whiskers are for $\mathrm{Q}_{3}+1.5 *\left(\mathrm{Q}_{3}-\mathrm{Q}_{1}\right)$ and $\mathrm{Q}_{1}-1.5 *\left(\mathrm{Q}_{3}-\mathrm{Q}_{1}\right)$, with $\mathrm{Q}_{1}$ and $\mathrm{Q}_{3}$ the first and third quartiles, respectively. Julian and Gregorian dates are in the left and right $y$-axes. b) Intensity, c) Magnitude and c) Duration of the events, the horizontal lines are for the $\mathrm{Q}_{1}, \mathrm{Q}_{2}$ (median) $\mathrm{y}_{3}$ quartiles.

los correspondientes en la cuenca del Tárcoles para la magnitud e intensidad, lo que podría asociarse con veranillos más secos en la cuenca del Tempisque. Dentro de los años más secos durante estos eventos se encuentran 1965, 1970, 1972, 1976, 1982, 1986, 2000 y 2001, nótese que no necesariamente coinciden con aquellos observados en la cuenca del Tárcoles. Para esta cuenca, la del Tempisque, el evento de mayor duración se observó para el año 1999.

El Cuadro 1, resume los valores de las medianas para las variables consideradas en ambas cuencas. Nótese que en general, los valores de la magnitud e intensidad de los eventos tienden a ser menores en la cuenca del Tempisque y que también tienden a ser de mayor duración, sin embargo, como se mencionó anteriormente, la variabilidad asociada con diferentes aspectos del veranillo tiende a ser alta. En ambas cuencas la mediana de la fecha del mínimo de los eventos se observó para el 21 de julio.

Los Cuadros 2 y 3 muestran las relaciones observadas entre la intensidad y la magnitud de los eventos (columnas) y las anomalías observadas en junio del índice Niño 3.4 (filas). No se encontraron relaciones estadísticamente significativas entre este índice climático y algún 

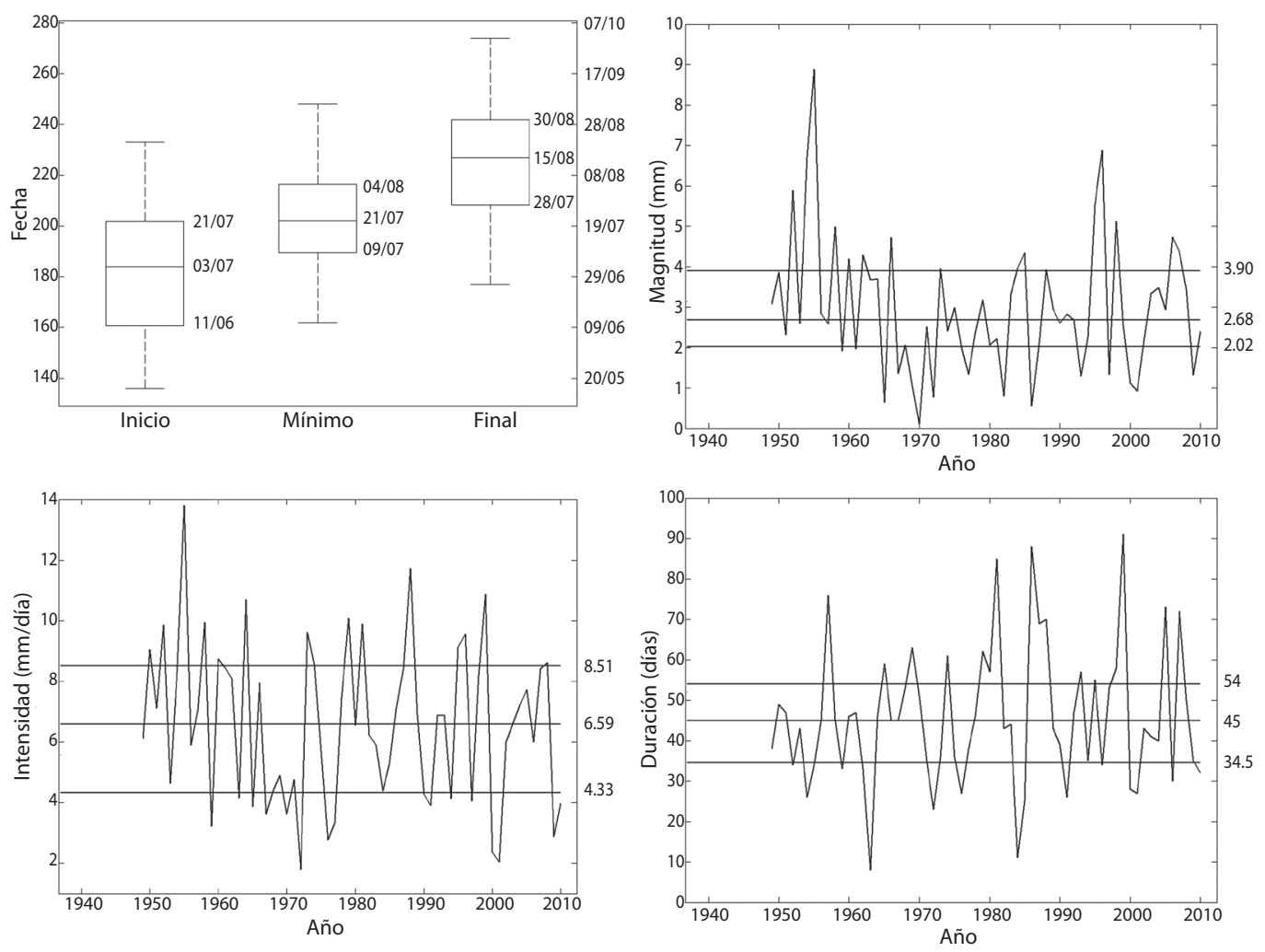

Fig. 4. Características del veranillo en la cuenca del Tempisque. a) Gráficos de cajas para el inicio, la fecha del mínimo y el final de los eventos. Los bigotes se calcularon como $Q_{3}+1.5 *\left(Q_{3}-Q_{1}\right)$ y $Q_{1}-1.5 *\left(Q_{3}-Q_{1}\right)$, con $Q_{1}$ y $Q_{3}$ el primer y tercer cuartil, respectivamente. En el eje $y$ se dan las fechas julianas (izquierda) y gregorianas (derecha). b) Intensidad, c) Magnitud y d) Duración de los eventos, las líneas horizontales son para los cuartiles $\mathrm{Q}_{1}, \mathrm{Q}_{2}$ (mediana) y $\mathrm{Q}_{3}$.

Fig. 4. Characteristics of the Mid Summer Drought at Tempisque basin. a) Box plots for the Star, Timing and End of the events. Whiskers are for $\mathrm{Q}_{3}+1.5 *\left(\mathrm{Q}_{3}-\mathrm{Q}_{1}\right)$ and $\mathrm{Q}_{1}-1.5 *\left(\mathrm{Q}_{3}-\mathrm{Q}_{1}\right)$, with $\mathrm{Q}_{1}$ and $\mathrm{Q}_{3}$ the first and third quartiles, respectively. Julian and Gregorian dates are in the left and right $y$-axes. b) Intensity, c) Magnitude and c) Duration of the events, the horizontal lines are for the $\mathrm{Q}_{1}, \mathrm{Q}_{2}$ (median) $\mathrm{y}_{3}$ quartiles.

CUADRO 1

Valores de las medianas para las características asociadas al veranillo en las cuencas estudiadas

TABLE 1

Median values for the Mid Summer Drought characteristics in the two river basin studied

\begin{tabular}{lcc} 
& \multicolumn{2}{c}{ Cuenca } \\
& Grande de Tárcoles & Tempisque \\
Inicio & 01 de Julio & 03 de julio \\
Fecha del mínimo & 21 de julio & 21 de julio \\
Final & 9 de agosto & 15 de agosto \\
Duración & 38 días & 45 días \\
Intensidad & $7,2 \mathrm{~mm} \mathrm{día}^{-1}$ & $6,6 \mathrm{~mm} \mathrm{día}^{-1}$ \\
Magnitud & $4,1 \mathrm{~mm}$ & $2,7 \mathrm{~mm}^{2}$ \\
\hline
\end{tabular}

otro aspecto asociado con el veranillo en las dos cuencas, ni entre alguno de los cinco aspectos considerados del veranillo y el índice de la Oscilación Multidecadal del Atlántico o AMO por sus siglas en inglés. El análisis de estas Tablas de Contingencia (Cuadros 2 y 3 ) arroja que en ambas cuencas, el escenario seco (BN) es el más probable para condiciones cálidas (AN) del índice Niño 3.4, tipo El Niño, mientras que los eventos menos severos del veranillo, agrupados en la categoría (AN), son más probables cuando se asocian con anomalías frías (BN) del índice Niño 3.4, tipo La Niña.

Nótese que al observarse la mayoría de las fechas del mínimo de los eventos del veranillo 


\section{CUADRO 2}

Probabilidades empíricas condicionales para la intensidad (arriba con $\mathrm{r}_{\mathrm{s}}=-0,44 * * *$ ) y la magnitud (abajo con $\mathrm{r}_{\mathrm{s}}=-0.50 * * *$ ) del veranillo en la cuenca del Grande de Tárcoles, dada una anomalía observada en junio del índice Niño 3.4. Los valores entre paréntesis son las frecuencias empíricas absolutas y las categorías usadas son bajo lo normal (BN), neutral (N)

y arriba de lo normal (AN). Los asteriscos denotan la significancia estadística,

donde $\alpha=0,01=>* * *, 0,05=>* *, 0.10=>*$. $\mathrm{r}_{\mathrm{s}}$ : correlación de Spearman

TABLE 2

Empirical conditional probabilities for the intensity (top, with $\mathrm{r}_{\mathrm{s}}=-0.44 * * *$ ) and the magnitude (bottom with $\mathrm{r}_{\mathrm{s}}=-0.50^{* * *}$ ) of the Mid Summer Drought in the Grande de Tarcoles river basin. Here, BN indicates the category below normal, $\mathrm{N}$ is normal, and AN denotes above normal. The asterisks indicates statistical significance level for $\alpha=0,01=>* * *, 0,05=>* *, 0,10=>* . \mathrm{r}_{\mathrm{s}}$ : Spearman correlation

\begin{tabular}{|c|c|c|c|c|}
\hline & & \multicolumn{3}{|c|}{ Intensidad } \\
\hline & & $\mathrm{BN}$ & $\mathrm{N}$ & AN \\
\hline & & $\left(\leq 6,4 \mathrm{~mm}\right.$ día $\left.^{-1}\right)$ & & $\left(\geq 8,3 \mathrm{~mm} \mathrm{día}{ }^{-1}\right)$ \\
\hline & $\mathrm{BN}\left(\leq-0,17^{\circ} \mathrm{C}\right)$ & $16(4)^{* * *}$ & $28(7)^{*}$ & $56(14)^{* * *}$ \\
\hline Niño 3.4 & $\mathrm{~N}$ & $21(5)^{*}$ & $50(12)^{* *}$ & $29(7)$ \\
\hline \multirow[t]{5}{*}{ Junio } & $\mathrm{AN}\left(\geq 0,42^{\circ} \mathrm{C}\right)$ & $64(16)^{* * *}$ & $20(5)^{*}$ & $16(4) * * *$ \\
\hline & & \multicolumn{3}{|c|}{ Magnitud } \\
\hline & & BN & $\mathrm{N}$ & AN \\
\hline & & $(\leq 3,3 \mathrm{~mm})$ & & $(\geq 4,8 \mathrm{~mm})$ \\
\hline & $\mathrm{BN}\left(\leq-0.17^{\circ} \mathrm{C}\right)$ & $12(3) * * *$ & $28(7)$ & $60(15)^{* * *}$ \\
\hline Niño 3.4 & $\mathrm{~N}$ & $29(7)$ & $50(12)^{* *}$ & $21(5)^{*}$ \\
\hline Junio & $\mathrm{AN}\left(\geq 0,42^{\circ} \mathrm{C}\right)$ & $60(15)^{* * *}$ & $20(5)^{*}$ & $20(5)^{* *}$ \\
\hline
\end{tabular}

CUADRO 3

Probabilidades empíricas condicionales para la intensidad (arriba con $\mathrm{r}_{\mathrm{s}}=-0,30 * *$ ) y la magnitud (abajo con $\mathrm{r}_{\mathrm{s}}=-0,41^{* * *}$ ) del veranillo en la cuenca del Tempisque, dada una anomalía observada en junio del índice Niño 3.4.

Los valores entre paréntesis son las frecuencias empíricas absolutas y las categorías usadas son bajo lo normal (BN), neutral $(\mathrm{N})$ y arriba de lo normal (AN). Los asteriscos denotan la significancia estadística, donde $\alpha=0,01=>* * *, 0,05=>* *, 0,10=>*$. $\mathrm{r}_{\mathrm{s}}$ : correlación de Spearman

TABLE 3

Empirical conditional probabilities for the intensity (top, with $r_{s}=-0,30 * *$ ) and the magnitude (bottom with $r_{s}=-0,41 * * *$ ) of the Mid Summer Drought in the Tempisque river basin. Here, BN indicates the category below normal, $\mathrm{N}$ is normal, and AN denotes above normal. The asterisks indicates statistical significance level for $\alpha=0,01=>* * *, 0,05=>* *, 0,10=>* . r_{\mathrm{s}}$ : Spearman correlation

\begin{tabular}{|c|c|c|c|c|}
\hline & & \multicolumn{3}{|c|}{ Intensidad } \\
\hline & & $\mathrm{BN}$ & $\mathrm{N}$ & $\mathrm{AN}$ \\
\hline & & $\left(\leq 5,1 \mathrm{~mm}\right.$ día $\left.^{-1}\right)$ & & $\left(\geq 8,0 \mathrm{~mm}\right.$ día $\left.^{-1}\right)$ \\
\hline & $\mathrm{BN}\left(\leq-0,23^{\circ} \mathrm{C}\right)$ & $24(5)^{*}$ & $28(6)$ & $48(10)^{* *}$ \\
\hline Niño 3.4 & $\mathrm{~N}$ & $33(7)$ & $29(6)$ & $38(8)$ \\
\hline \multirow[t]{5}{*}{ Junio } & $\mathrm{AN}\left(\geq 0,49^{\circ} \mathrm{C}\right)$ & $43(9)$ & $43(9)$ & $14(3) * * *$ \\
\hline & & \multicolumn{3}{|c|}{ Magnitud } \\
\hline & & $\mathrm{BN}$ & $\mathrm{N}$ & AN \\
\hline & & $(\leq 2,2 \mathrm{~mm})$ & & $(\geq 3,4 \mathrm{~mm})$ \\
\hline & $\mathrm{BN}\left(\leq-0,23^{\circ} \mathrm{C}\right)$ & $10(2) * * *$ & $43(9)$ & $47(10)^{* *}$ \\
\hline Niño 3.4 & $\mathrm{~N}$ & $33(7)$ & $29(6)$ & $38(8)$ \\
\hline Junio & $\mathrm{AN}\left(\geq 0,49^{\circ} \mathrm{C}\right)$ & $57(12)^{* * *}$ & $29(6)$ & $14(3)^{* * *}$ \\
\hline
\end{tabular}


en el mes de julio, los resultados de los Cuadros 2 y 3 sugieren un esquema de tipo predictivo sobre la intensidad y la magnitud de los mismos. Debido a que algunas instituciones como el Instituto Internacional de Investigación para el Clima y la Sociedad (IRI, por sus siglas en inglés) realizan o compilan la predicción estacional de las anomalías del índice Niño
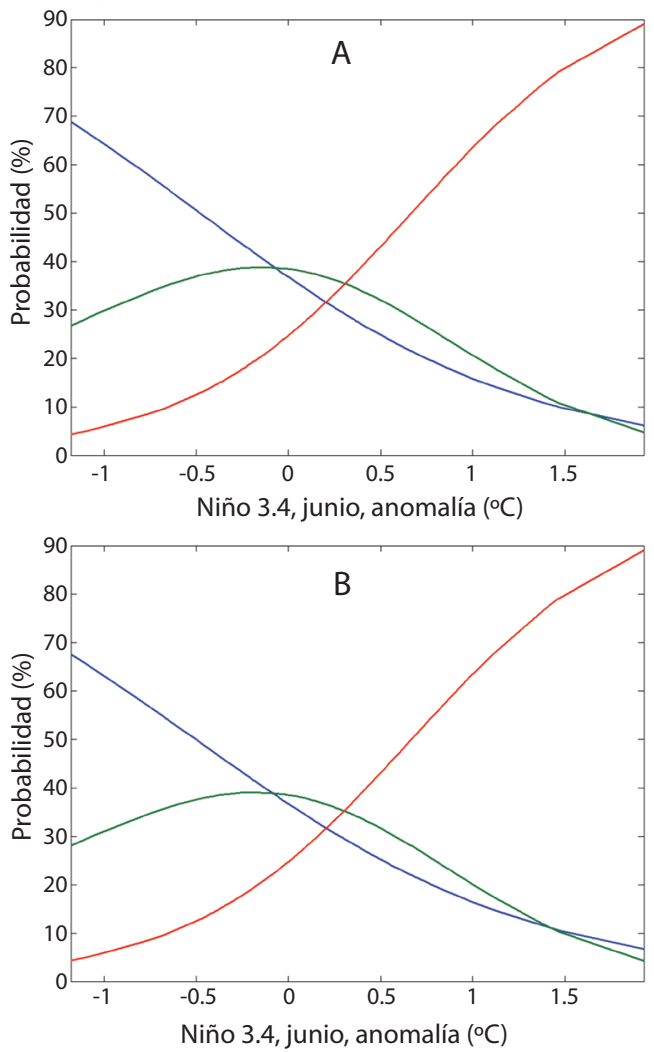

Fig. 5. Modelos logit ajustados de acuerdo a la Ec. 1, para la a) Intensidad y b) Magnitud del veranillo en la cuenca del Grande de Tárcoles, con $x$ dado por la anomalía observada en el índice Niño 3.4 durante el mes de junio. La línea azul, verde y roja son para la probabilidad de los escenarios AN, N y BN, respectivamente de los modelos $y_{\mathrm{AN}}, y_{\mathrm{N}}$ y $y_{\mathrm{BN}}$, presentados en el Cuadro 4 .

Fig. 5. Logit models fixed according to Eq. 1 for the Mid Summer Drought a) Intensity and b) Magnitude at the Grande de Tarcoles basin, with $x$ representing the June observed anomaly in the Niño 3.4 index. Blue, green and red line are for the probability of the $\mathrm{AN}, \mathrm{N}$ and $\mathrm{BN}$ scenarios, respectively of the models $y_{\mathrm{AN}}, y_{\mathrm{N}}$ and $y_{\mathrm{BN}}$, showed in Table 4.
3.4 (http://www.cpc.ncep.noaa.gov/products/ analysis_monitoring/enso_advisory, última visita 24/06/2013), se pueden realizar escenarios de probabilidad sobre las condiciones $\mathrm{BN}, \mathrm{N}$ y $\mathrm{AN}$ de la intensidad y magnitud del veranillo, dada una anomalía observada en el índice Niño 3.4. Estas relaciones se muestran en las Figs. 5 y 6, por medio del uso de modelos
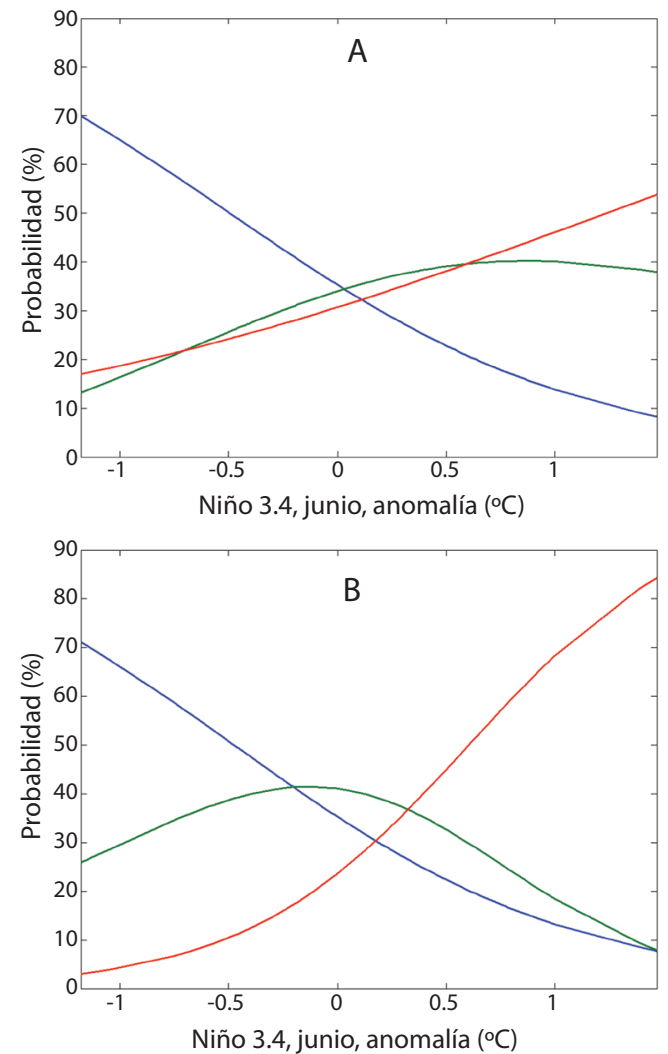

Fig. 6. Modelos logit ajustados de acuerdo a la Ec. 1, para la a) Intensidad y b) Magnitud del veranillo en la cuenca del Tempisque, con $x$ dado por la anomalía observada en el índice Niño 3.4 durante el mes de junio. La línea azul, verde y roja son para la probabilidad de los escenarios AN, $\mathrm{N}$ y BN, respectivamente de los modelos $y_{\mathrm{AN}}, y_{\mathrm{N}}$ y $y_{\mathrm{BN}}$, presentados en el Cuadro 4.

Fig. 6. Logit models fixed according to Eq. 1 for the Mid Summer Drought a) Intensity and b) Magnitude at the Tempisque basin, with $x$ representing the June observed anomaly in the Niño 3.4 index. Blue, green and red line are for the probability of the $\mathrm{AN}, \mathrm{N}$ and $\mathrm{BN}$ scenarios, respectively of the models $y_{\mathrm{AN}}, y_{\mathrm{N}}$ and $y_{\mathrm{BN}}$, showed in Table 4. 
CUADRO 4

Coeficientes de los modelos logit ajustados de acuerdo con la Ec. 1, para obtener las curvas de probabilidad de los escenarios arriba de lo normal ( $\mathrm{AN}$ ) y bajo lo normal $(\mathrm{BN})$ en las cuencas Grande de Tárcoles (arriba) y Tempisque (abajo), ver Figs. 5 y 6. Los valores de $z$ representan el coeficiente obtenido dividido entre su error estándar

TABLE 4

Logit model coefficients obtained according with Eq. 1, for the probability curves associated with the above and below (AN and BN) normal scenarios and for the Grande de Tarcoles (top) and Tempisque (bottom) river basins, see Figs. 5 and 6 . The $z$ values represent the coefficient divided by its standard error

\begin{tabular}{lcccc} 
Modelo, Tárcoles & $\beta_{0}$ & $\beta_{1}$ & $z_{0}$ & $z_{1}$ \\
Intensidad, $y_{\mathrm{AN}}$ & $-0,54$ & $-1,13$ & $-2,09$ & $-2,43$ \\
Intensidad, $y_{\mathrm{BN}}$ & $-1,11$ & 1,67 & $-3,50$ & 3,19 \\
Magnitud, $y_{\mathrm{AN}}$ & $-0,54$ & $-1,08$ & $-2,10$ & $-2,35$ \\
Magnitud, $y_{\mathrm{BN}}$ & $-1,11$ & 1,67 & $-3,50$ & 3,19 \\
Modelos, Tempisque & $\beta_{0}$ & $\beta_{1}$ & $z_{0}$ & $z_{1}$ \\
Intensidad, $y_{\mathrm{AN}}$ & $-0,61$ & $-1,23$ & $-2,14$ & $-2,44$ \\
Intensidad, $y_{\mathrm{BN}}$ & $-0,81$ & 0,66 & $-2,80$ & 1,45 \\
Magnitud, $y_{\mathrm{AN}}$ & $-0,60$ & $-1,28$ & $-2,14$ & $-2,51$ \\
Magnitud, $y_{\mathrm{BN}}$ & $-1,17$ & 1,94 & $-3,26$ & 3,24 \\
\hline
\end{tabular}

logit. El Cuadro 4 lista los coeficientes ajustados para los escenarios BN y AN. Se observa que el único coeficiente no significativo al $95 \%$ $(|z|>1,96)$ fue el de la intensidad en la cuenca del Tempisque para el escenario BN. Todos los modelos ajustados muestran que el escenario más probable para anomalías positivas (negativas) en el índice Niño 3.4 es el BN (AN) en la intensidad y magnitud del veranillo en ambas cuencas.

La Fig. 7 muestra los principales impactos asociados al periodo de veranillo recopilados de los Boletines Meteorológicos Mensuales del IMN. Las categorías que mostraron la mayor cantidad de impactos fueron déficit de lluvias y aguaceros fuertes (Fig. 7a). Esta aparente contradicción, se aclara al graficarlos espacialmente. La vertiente del Pacífico es la que reporta mayor cantidad de impactos asociados a déficit de lluvia junto con temperaturas por encima del promedio y la del Caribe es la región que más impactos reporta asociados a aguaceros fuertes junto con inundaciones (Fig. 7b). Lo anterior concuerda con el escenario esperado de un incremento de los vientos alisios con componente del este sobre Costa Rica. Nótese que los impactos debido a vientos fuertes se distribuyeron en forma más homogénea sobre el país.

\section{DISCUSIÓN}

La metodología empleada permitió la caracterización del veranillo en dos de las principales cuencas de la vertiente del Pacífico de Costa Rica, a saber, río Grande de Tárcoles y río Tempisque, ambas con problemas en la gestión del recurso hídrico. Los aspectos considerados para esta caracterización comprendieron el inicio, duración y final del evento, la fecha del mínimo, su intensidad y magnitud. Al igual que lo observado por Ramírez (1983), Magaña et al. (1999), Amador (2008) y Karnauskas et al. (2013), estos aspectos mostraron una variabilidad interanual, que estos autores relacionan con la variabilidad de la actividad del monzón de América del Norte, la Corriente en Chorro de Bajo Nivel del Caribe y la Alta subtropical del Atlántico Norte, principalmente, además de la variabilidad en la intensificación de los vientos alisios durante julio y agosto, junto con el forzamiento orográfico de las montañas en buena parte de América Central, provocando una variabilidad en el máximo de precipitación a lo largo de la costa del Caribe y en el mínimo en la del Pacífico.

Los eventos del veranillo en ambas regiones tienden a iniciar a principios de julio con 

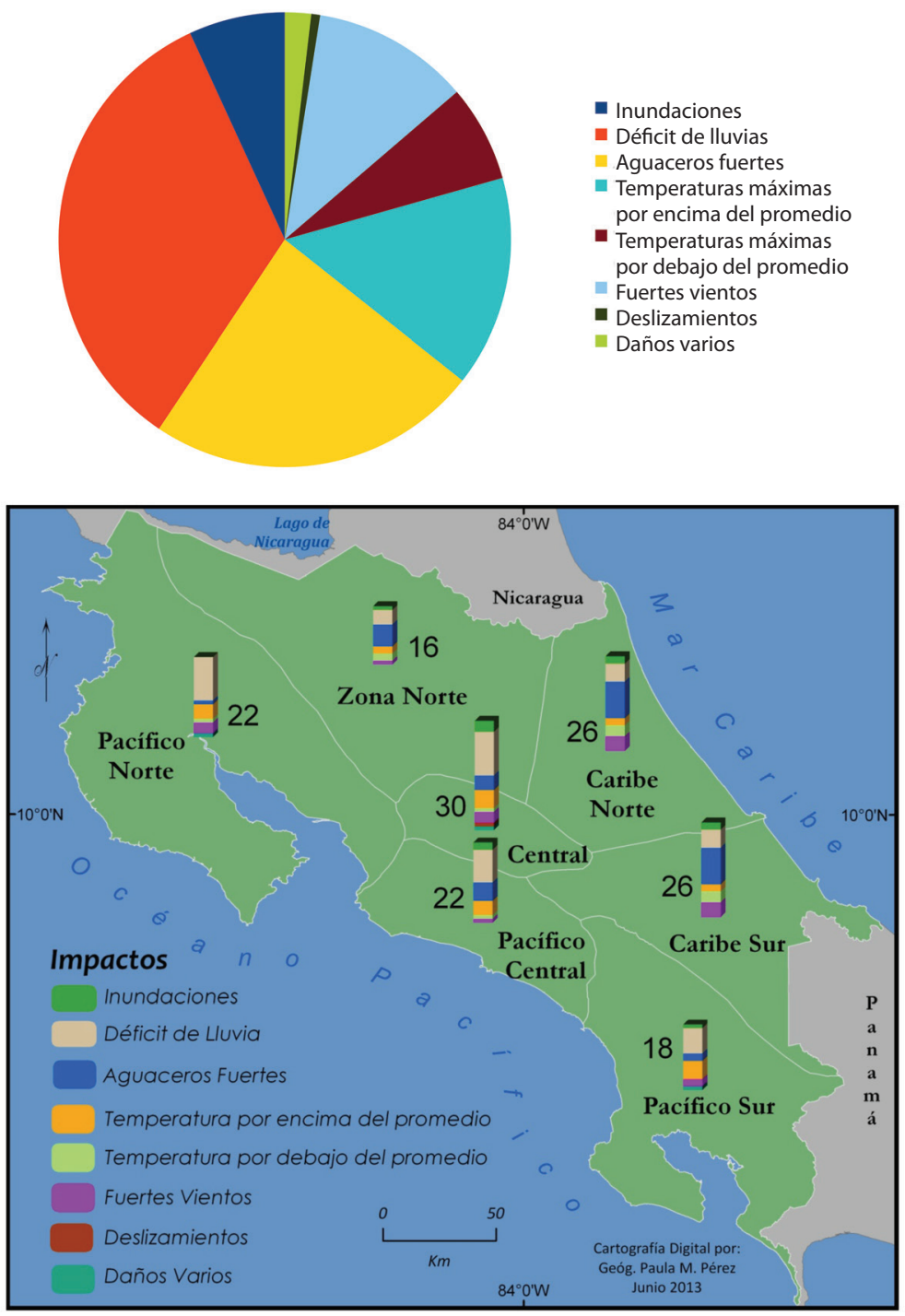

Fig. 7. a) Distribución porcentual de los impactos climáticos reportados en los Boletines Meteorológicos Mensuales, elaborados por el Instituto Meteorológico Nacional de Costa Rica, durante los años 1977, 1982, 1986, 1993, 2000, 2001, 2003, 2009 y 2012. b) Distribución espacial de los impactos mostrados en a), de acuerdo a las regiones climáticas de Costa Rica.

Fig. 7. a) Percentage distribution of climate impacts reported in the Monthly Meteorological Bulletins elaborated by the Costa Rican National Meteorological Institute for the years 1977, 1982, 1986, 1993, 2000, 2001, 2003, 2009 and 2012. b) Spatial distribution of the impacts showed in a), according to the climate regions of Costa Rica.

mínimos ubicados alrededor del 21 de julio, finalizando generalmente en los primeros quince días de agosto, lo que coincide con el trabajo previo de Ramírez (1983). En la cuenca del Grande de Tárcoles, estos eventos duraron alrededor de 38días, con precipitaciones promedio asociadas de $7,2 \mathrm{~mm} \mathrm{día}^{-1}$ y $4,1 \mathrm{~mm}$ de lluvia en sus mínimos. En la cuenca del Tempisque estos valores fueron de 45 días, $6,6 \mathrm{~mm}$ día $^{-1}$ y $2,7 \mathrm{~mm}$, respectivamente. Observándose eventos más prolongados y más secos en la del Tempisque. 
El análisis de Tablas de Contingencia mostró que en ambas cuencas, el escenario seco (BN) es el más probable para condiciones cálidas (AN) del índice Niño 3.4, tipo El Niño, mientras que los eventos menos severos del veranillo, agrupados en la categoría (AN), son más probables cuando se asocian con anomalías frías (BN) del índice Niño 3.4, tipo La Niña. En ambos casos, los escenarios seco-La Niña y húmedo-El Niño, fueron muy poco probables.

Por medio del uso de modelos logit, se pueden realizar escenarios de probabilidad sobre las condiciones $\mathrm{BN}, \mathrm{N}$ y $\mathrm{AN}$ de la intensidad y magnitud del veranillo, dada una anomalía observada en el índice Niño 3.4 en el mes de junio. Los modelos ajustados mostraron que el escenario más probable para anomalías positivas (negativas) en el índice Niño 3.4 es el BN (AN) en la intensidad y magnitud del veranillo en ambas cuencas, o sea, aquellas categorías asociadas con los veranillos más (menos) secos. Al observarse la mayoría de las fechas del mínimo de los eventos del veranillo en el mes de julio, estos resultados tienen potencial de uso en predicción estacional. Esta predicción puede ser utilizada en la gestión de los ecosistemas de gran importancia económica para Costa Rica, como por ejemplo el humedal de Palo Verde (Moreno-Díaz, Choden, Floquet \& Mongbo, 2011), incluida su asociación con la incidencia de incendios forestales en la temporada seca siguiente (Alfaro, Fernández \& Connell, 1999).

Las categorías que mostraron la mayor cantidad de impactos climáticos asociados con el veranillo fueron el déficit de lluvias y aguaceros fuertes. Se observó que la vertiente del Pacífico es la que reporta mayor cantidad de impactos asociados a déficit de lluvia junto con temperaturas por encima del promedio y la del Caribe es la región que más impactos reporta asociados a aguaceros fuertes junto con inundaciones. Lo anterior concuerda con el escenario esperado de un incremento del viento alisio del este sobre Costa Rica (Ramírez 1983; Magaña et al., 1999; Amador, 2008 y Karnauskas et al., 2013). Los impactos debido a vientos fuertes se distribuyeron en forma más homogénea sobre el país.

\section{AGRADECIMIENTOS}

El estudio se realizó dentro del marco de los siguientes proyectos de la Vicerrectoría de Investigación de la Universidad de Costa Rica: 808-B2-400 (apoyado por la Fundación Costa Rica x Siempre y la Agencia Alemana de Cooperación GiZ a través del Proyecto BIOMARC), 808-A9-180, 805-A8-606 (apoyado por FI\&F), 805-B3-600 (apoyo del fondo de estímulo UCR), 805-A9-224 (apoyo del fondo de estímulo UCR), 805-B0-402 (apoyado por CORBANA), 805-A7-002 (apoyado por GEOF 02 2013-IPGH, 805-A9-523 (apoyado por CSUCA-ASDI) y CRN2050-IAI), UCR. A Paula Pérez y Elsie Troyo por su ayuda con el procesamiento de la información de los boletines del IMN.

\section{RESUMEN}

La región de los Mares Intra-Americanos está caracterizada por fenómenos climáticos raramente observados en regiones tropicales, uno de ellos es el llamado "veranillo" o "canícula". Sobre la vertiente del Pacífico de América Central, el ciclo anual de la precipitación está caracterizado por dos máximos, uno en junio y el otro, mayor que el primero, en setiembre-octubre, con una época seca de noviembre a mayo y un periodo corto de reducción de precipitaciones durante julio-agosto (veranillo o canícula). Se estudiaron siete registros diarios de precipitación, pertenecientes las estaciones ubicadas en dos cuencas importantes de Costa Rica, río Grande de Tárcoles y río Tempisque, para caracterizar el veranillo en el periodo 1937-2012. Dentro de los aspectos considerados, está el inicio, duración y final del evento, la fecha del mínimo, así como su intensidad y magnitud. Se exploró luego la modulación de estos aspectos debido a fuentes de variabilidad climática tales como el Pacífico Ecuatorial del Este, en donde se observó que condiciones cálidas (frías) del índice Niño 3.4, tienden a estar relacionadas con eventos más secos (más lluviosos) de veranillos.

Palabras clave: Veranillo, Canícula, Variabilidad Climática, Tempisque, Grande de Tárcoles, predicción climática estacional, análisis categórico, América Central

\section{REFERENCIAS}


Alfaro, E.J., Soley, F.J. \& Enfield, D. (2003). Uso de una Tabla de Contingencia para Aplicaciones Climáticas (Use of a Contingency Table for Climatic Applications). Guayaquil, Ecuador: ESPOL-FUNDESPOL.

Alfaro, E.J. \& Soley, F.J. (2009). Descripción de dos métodos de rellenado de datos ausentes en series de tiempo meteorológicas. Rev. Mate.: Teor. Aplic.,16, 59-74.

Alfaro, R., Fernández, W. \& Connell, B. (1999). Detection of the forest fires of April 1997 in Guanacaste, Costa Rica, using GOES-8 images. Int. J. Remote Sensing, 20, 1189-1195.

Amador, J. (2008). The Intra-Americas Seas Low-Level Jet (IALLJ): Overview and future research. Ann. N.Y. Acad. Sci., 1146, 153-188.

Amador, J.A., Alfaro, E.J., Lizano, O.G. \& Magaña, V.O. (2006). Atmospheric forcing in the Eastern Tropical Pacific: A review. Progr. Oceanogr., 69, 101-142.

Echeverría-Bonilla, J. (2004). Estudio de caso cuenca Tempisque, Costa Rica. Informe técnico. Estrategia para la Gestión Integrada de los Recursos Hídricos en Costa Rica. Plan Nacional de Gestión Integrada del Recurso Hídrico. San José, Costa Rica: Ministerio del Ambiente y Energía. (Consultado: 01 de julio de 2013, http://www.drh.go.cr/textos/estrategia/)

Espinoza, C.E. \& Villalta, R.A. (2004). Estudio de caso sobre la contaminación de la cuenca de los ríos virilla y grande de tárcoles (cuenca 24). Documento técnico. Estrategia para la Gestión Integrada de los Recursos Hídricos en Costa Rica. Plan Nacional de Gestión Integrada del Recurso Hídrico. San José, Costa Rica: Ministerio del Ambiente y Energía. (Consultado: 01 de julio de 2013, http://www.drh.go.cr/ textos/estrategia/)

INEC (2012). X censo nacional de población y VI de vivienda 2011: Características sociales y demográficas. Tomo I. San José, Costa Rica: Instituto Nacional de Estadística y Censos.
Karnauskas, K.B., Giannini, A., Seager, R. \& Busalacchi, A.J. (2013). A simple mechanism for the climatological midsummer drought along the Pacific coast of Central America. Atmósfera, 26, 261-281.

Magaña, V.O., Amador, J.A. \& Medina, S. (1999). The midsummer drought over Mexico and Central America. J. Clim., 12, 1577-1588.

Moreno-Díaz, M.L., Choden, S., Floquet, A. \& Mongbo, R.L. (2011). Protected Areas - not just for biodiversity Conservation: The contributions of protected areas to the economic and social development in Bhutan, Costa Rica and Benin. Heredia, Costa Rica: UNA, CINPE, CEBEDES, NCD, Zeta Servicios Gráficos S.A.

Nakaegawa, T., Kitoh, A., Ishizaki, Y., Kusunoki, S. \& Murakami, H. (2013). Caribbean low-level jets and accompanying moisture fluxes in a global warming climate projected with CMIP3 multi-model ensemble and fine-mesh atmospheric general circulation models. Int. J. Climatol., doi: 10.1002/joc.3733.

Ramírez, P. (1983). Estudio Meteorológico de los Veranillos en Costa Rica. Informe Técnico. Nota de investigación No 5. San José, Costa Rica: Instituto Meteorológico Nacional, Ministerio de Agricultura y Ganadería.

Soley, F.J. (1994). Suavizamiento de series cronológicas geofísicas con ruido blanco y rojo aditivo. Rev. Geofis., 41, 33-58.

Taylor, M. A., Enfield, D. B. \& Chen, A. A. (2002). Influence of the tropical Atlantic versus the tropical Pacific on Caribbean rainfall, J. Geophys. Res., 107, doi:10.1029/2001JC001097.

Wang, C. \& Enfield, D. B. (2003). A further study of the tropical western hemisphere warm pool. J. Clim., 16, 1476-1493.

Wilks, D. (2006). Statistical Methods in the Atmospheric Sciences (2da. ed.). San Diego, California: Academic. 
